\title{
Nonaxisymmetric Neutral Modes in Relativistic Disks
}

\author{
Mike J. Cai \\ Institute of Astronomy and Astrophysics, Academia Sinica \\ P.O. Box 23-141, Taipei 106, Taiwan, R.O.C. \\ mike@asiaa.sinica.edu.tw \\ Frank H. Shu \\ Department of Physics, National Tsing-Hua University \\ Hsinchu 300, Taiwan, Republic of China \\ shu@mx.nthu.edu.tw
}

\begin{abstract}
We perform a linear stability analysis of the axisymmetric, relativistic, selfsimilar isothermal disk against non-axisymmetric perturbations. Two sets of neutral modes are discovered. The first set corresponds to marginally unstable perturbations driven by gravitational radiation, and the other signals the onset of bifurcation to non-axisymmetric equilibrium solutions to the Einstein equations.
\end{abstract}

\section{Introduction}

Cai \& Shu (2002) put forward the hypothesis that relativistic disks may ultimately play as important a role in astrophysics as their spherical counterparts. There is evidence that supermassive blackholes in quasars were formed during the early stages of galaxy formation, where the major contributor to the mass-energy density was gas rather than stars. The dynamics of gas is highly dissipative, so it is easier for the gas to lose significant amounts of energy to reach the desired compactness for general relativistic effects to be important. More problematic is how to get rid of excess angular momentum if it is present initially.

The pioneering work of Bardeen \& Wagoner (1971) on uniformly rotating disks showed that with some assumed angular momentum loss, a Kerr blackhole may result in the collapse of such disks. However, as Mestel (1963) pointed out in a Newtonian context, there are astrophysical reasons to think that a disk specified by constant linear rotation velocity $v$ is more realistic than one which has constant angular velocity. Through bar formation and 
spiral density waves (for a review, e.g., see Shu et. al. 2000), such differentially rotating disks possess natural mechanisms for the outward transport of angular momentum and the inward transport of mass that would promote, in the relativistic regime, blackhole formation at the center.

The first step in a systematic theoretical study of this possibility is to construct fully relativistic, self-similar, rotating, flattened solutions. Lynden-Bell \& Pineault (1978) performed such a pioneering analysis, but only in the cold limit. To suppress well-known axisymmetric instabilities that would fragment the disk into rings, it is necessary to include partial support from isothermal pressure. If the pressure is exerted isotropically in three directions, the result (Cai \& Shu 2003) is the relativistic generalization of the singular isothermal toroids (SITs) found by Toomre (1982) and Hayashi et. al. (1982). A useful approximation when such states become sufficiently flattened by rotation is to ignore the thermal dispersive speed in the vertical direction, while retaining it in the horizontal directions. In this approximation SITs become completely flattened, singular isothermal disks (SIDs), whose equilibrium properties in the relativistic regime were studied by Cai \& Shu (2002). These solutions are infinite in extent, possess infinite total mass, and contain a naked singularity at the origin (a "baby blackhole" with vanishingly small mass).

The formal approximation of highly flattened configurations is satisfied for SITs only when the Mach number $M \equiv v / \sqrt{\gamma} \gg 1$. Nevertheless, , even if $M \sim 1$, Cai \& Shu (2003) found that the critical condition under which the sequence of equilibria terminates as a function of $M$ is nearly identical whether the pressure is exerted in three dimensions (SITs) or two (SIDs). The insensitivity of crucial properties of the equilibria to the assumption of infinitesimal thickness will hopefully carry over to the analysis of their stability.

On dimensional grounds, if the disk becomes gravitationally unstable to overall gravitational collapse (a possibility if the disk is sufficiently slowly rotating), the mass of the baby black hole will grow linearly in time as a result of axisymmetric collapse. In the analogous problem of the collapse of relativistic singular isothermal sphere (SIS), Cai \& Shu (2004) have shown that the growth of a black hole with finite mass introduces a (spherically symmetric) horizon which covers up the singularity. It is intriguing to ask whether such singularity in the case of a collapsing, relativistic SID will remain naked if the requirement of axial symmetry is relaxed. In order to answer this question, one must first construct relativistic SIDs that are non-axisymmetric states of equilibria. One of the goals of the present paper is to make a start on this problem, by finding the points of non-axisymmetric bifurcation along the sequence of axisymmetric SIDs, thereby generalizing the Newtonian work of Syer \& Tremaine (1996) and Galli et. al. (2001).

A feature with no Newtonian analog appears with the completion of the analysis: the 
appearance of a secular instability which afflicts all relativistically rotating disks. This instability, associated classically with the name Rossby (so the corresponding perturbations are called R-modes), arises because general relativity admits the radiation of angular momentum (and energy) by gravitational waves (Chandrasekhar 1970a,b). Basically, if the rotation of the underlying axisymmetric state is high enough, a counter-rotating disturbance appears corotating to an inertial observer. Such disturbances have negative angular momentum density in the local rest frame of the disk. Since gravitational radiation carries away positive angular momentum, the amplitude of the R-mode perturbation grows in time.

The phenomenon renders, in some sense, all astrophysically rotating systems potentially unstable to spin-down on a gravitational-radiation time scale. Whether the R-mode instability competes with the gravitational torques associated with barlike deformations or spiral density waves remains a problem for future study. The self-similar models and techniques used in the present paper are capable only of determining the criterion for the onset of secular instabilities, and not their growth rates and evolution into the nonlinear regime. Thus, modifications are still required for application to astrophysically realistic circumstances, where the origin does not contain a singularity from the start, and where spacetime at infinity is flat.

In this paper, we restrict our study of the stability of relativistic SIDs to non-axisymmetric perturbations with the same scale-free character as the equilibrium state (i.e., with the same power-law radial dependence). In the nomenclature of Syer \& Tremaine (1996), we consider only aligned perturbations, and no spiral disturbances. In section 2, we review the basic

properties of axisymmetric SIDs. In section 3, we develop the mathematical formulation of the stability analysis, including the metric and matter perturbation. In section 4, the equations are solved in the Newtonian limit, and the result is compared to Shu et. al. (2000). In section 6 , the perturbation equations are solved in the full relativistic context, and we offer physical interpretation of the results.

\section{Review of Axisymmetric Disk Solution}

Start out with a self-similar axisymmetric metric

$$
d s^{2}=-r^{2 n} e^{N} d t^{2}+r^{2} e^{2 P-N}\left(d \phi-r^{n-1} e^{N-P} Q d t\right)^{2}+e^{Z-N}\left(d r^{2}+r^{2} d \theta^{2}\right),
$$

where $N, P, Q, Z$ are functions of $\theta$ and $n$ is a constant measuring the strength of the gravitational field. For numerical convenience, we have chosen the equatorial plane to be at some polar angle $\theta_{0}$, which is determined as an eigenvalue of the problem. The locally nonrotating observer (LNRO) defines an orthonormal tetrad frame analogous to the inertial 
frame:

$$
\begin{aligned}
e_{(0)}{ }^{\mu} & =\left(r^{-n} e^{-\frac{1}{2} N}, r^{-1} Q e^{\frac{1}{2} N-P}, 0,0\right) \\
e_{(1)}{ }^{\mu} & =\left(0, r^{-1} e^{\frac{1}{2} N-P}, 0,0\right) \\
e_{(2)}{ }^{\mu} & =\left(0,0, e^{\frac{1}{2}(N-Z)}, 0\right) \\
e_{(3)}{ }^{\mu} & =\left(0,0,0, r^{-1} e^{\frac{1}{2}(N-Z)}\right) .
\end{aligned}
$$

We look for solutions to the Einstein field equations with a disk matter source described by a constant linear rotation velocity and a two-dimensional isotropic pressure. In the frame of a LNRO, the stress-energy tensor is taken to be

$$
\begin{aligned}
& T_{(0)(0)}=\frac{\varepsilon+p_{\phi} v^{2}}{1-v^{2}}, \quad T_{(0)(1)}=-\frac{\left(\varepsilon+p_{\phi}\right) v}{1-v^{2}}, \\
& T_{(1)(1)}=\frac{p_{\phi}+\varepsilon v^{2}}{1-v^{2}}, \quad T_{(2)(2)}=p_{r},
\end{aligned}
$$

where $\varepsilon \propto \delta\left(\theta-\theta_{0}\right)$ and $p_{\phi}=p_{r}=\gamma \varepsilon$. Define

$$
\Theta=(1+n) \theta, \quad{ }^{\prime}=\frac{d}{d \Theta}, \quad \tilde{\varepsilon}=8 \pi \frac{\varepsilon}{1+n} r^{2} e^{Z_{0}-N_{0}}, \quad \Delta=\delta\left(\Theta-\Theta_{0}\right) .
$$

After some algebra, part of the Einstein equations are cast into a set of dynamic equations

$$
\begin{aligned}
N^{\prime \prime}= & -N^{\prime} P^{\prime}-\frac{2 n}{1+n}+Q^{2} F^{2}+Q^{2}\left(\frac{1-n}{1+n}\right)^{2}+\tilde{\varepsilon}\left[\frac{1+2 \gamma+v^{2}}{1-v^{2}}\right] \Delta, \\
P^{\prime \prime}= & -P^{\prime 2}-1+\tilde{\varepsilon} \gamma \Delta, \\
Q^{\prime \prime}= & -Q^{\prime} P^{\prime}+Q\left[\left(1-Q^{2}\right)\left(\frac{1-n}{1+n}\right)^{2}+\left(N^{\prime}-P^{\prime}\right)^{2}-Q^{2} F^{2}\right] \\
& -\tilde{\varepsilon}(1+\gamma) \frac{2 v+Q+Q v^{2}}{1-v^{2}} \Delta, \\
Z^{\prime \prime}= & -Z^{\prime} P^{\prime}+2 Q^{2}\left(\frac{1-n}{1+n}\right)^{2}-\frac{4 n^{2}}{(1+n)^{2}}+Q^{2} F^{2}+2 \tilde{\varepsilon}\left[\frac{v^{2}+\gamma}{1-v^{2}}\right] \Delta,
\end{aligned}
$$

where $F=N^{\prime}+\log Q^{\prime}-P^{\prime}$. The rest of the field equations and the equation of motion form a set of constraint equations

$$
\begin{aligned}
& Q\left(\Theta_{0}\right) v(1-n)(1+\gamma)+\gamma+v^{2}-n(1+\gamma)=0 \\
& Z^{\prime}-\frac{2 n}{1+n} N^{\prime}-Q^{2} \frac{1-n}{1+n} F=0 \\
& Q^{2}\left\{F^{2}-\left(\frac{1-n}{1+n}\right)^{2}\right\}+2 \cot \Theta Z^{\prime}-N^{\prime 2}+\frac{4 n^{2}}{(1+n)^{2}}=0
\end{aligned}
$$


Due to the contracted Bianchi identity, two of these equations are redundant, which were used for a numerical consistency check. For detailed discussion on the properties of these solutions, the readers are invited to refer to Cai \& Shu (2002).

\section{Perturbed Configuration}

We will use the Eulerian description for the non-axisymmetric modes. The perturbation in the metric is

$$
\delta g_{\mu \nu}=h_{\mu \nu}, \quad\left|\frac{h_{\mu \nu}}{g_{\alpha \beta}}\right| \ll 1 .
$$

We define the change in the contravariant components of the metric as

$$
\delta g^{\mu \nu}=-h^{\mu \nu}=-h_{\alpha \beta} g^{\alpha \mu} g^{\beta \nu}
$$

so that ${ }^{1}$

$$
\left(g^{\mu \nu}+\delta g^{\mu \nu}\right)\left(g_{\nu \rho}+\delta g_{\nu \rho}\right)=\delta_{\rho}^{\mu}+O\left(h^{2}\right) .
$$

Notice that $h_{\mu \nu}$ is not a tensor with respect to the unperturbed metric, and its projection onto LNRO is not a Lorentz scalar. Thus the directional derivatives of $h_{(a)(b)}$ will involve more than the usual Ricci rotation coefficients, which destroys the simplicity of the tetrad formalism. As a result, we will compute in the coordinate frame whenever derivatives are involved. However, the tetrad frame defined by (2-2) does offer a clean separation of $r$ from the other coordinates, so we shall project our results onto LNRO after the derivatives have been taken.

The change in the Ricci tensor reads (see, e.g., Wald 1984)

$$
\delta R_{\mu \nu}=\frac{1}{2}\left(h_{\mu ; \nu \alpha}^{\alpha}+h_{\nu ; \mu \alpha}^{\alpha}-h_{\mu \nu ; \alpha}^{\alpha}-h_{; \mu \nu}\right)
$$

where the raising and lowering of indices are done with the unperturbed metric. Instead of computing the Einstein tensor, we work out the trace-reversed stress-energy tensor. Taking the direct variation of the stress-energy tensor, we have

$$
\delta\left(T_{\mu \nu}-\frac{1}{2} g_{\mu \nu} T\right)=\delta T_{\mu \nu}-\frac{1}{2} h_{\mu \nu} T-\frac{1}{2} g_{\mu \nu} \delta T^{\alpha \beta} g_{\alpha \beta}-\frac{1}{2} g_{\mu \nu} T^{\alpha \beta} h_{\alpha \beta} .
$$

There is one subtlety in writing down this expression, due to the non-tensorial nature of the metric variations $h_{\mu \nu}$. Explicitly,

$$
\delta T_{\mu \nu}=\delta T^{\alpha \beta} g_{\alpha \mu} g_{\beta \nu}+T_{\mu}^{\alpha} h_{\alpha \nu}+T_{\nu}^{\alpha} h_{\alpha \mu} \neq \delta T^{\alpha \beta} g_{\alpha \mu} g_{\beta \nu}
$$

\footnotetext{
${ }^{1}$ It is unfortunate that we have two $\delta$ symbols here - one denoting Eulerian change and the other denoting the Kronecker-Delta function. There should be little confusion from context, however.
} 
To avoid such confusion, we shall adopt the convention that only contravariant components $T^{\alpha \beta}$ are varied ${ }^{2}$. So the Einstein field equation reads

$$
\begin{aligned}
& h_{\mu ; \nu \alpha}^{\alpha}+h^{\alpha}{ }_{\nu ; \mu \alpha}-h_{\mu \nu ; \alpha}{ }^{\alpha}-h_{; \mu \nu} \\
= & 8 \pi\left(2 \delta T^{\alpha \beta} g_{\alpha \mu} g_{\beta \nu}+2 T_{\mu}{ }^{\alpha} h_{\alpha \nu}+2 T_{\nu}{ }^{\alpha} h_{\alpha \mu}-h_{\mu \nu} T-g_{\mu \nu} \delta T^{\alpha \beta} g_{\alpha \beta}-g_{\mu \nu} T^{\alpha \beta} h_{\alpha \beta}\right) .
\end{aligned}
$$

\subsection{Gauge Choice}

Let's consider perturbations with time and angular dependence $e^{i m \phi-i \omega t}$. On dimensional grounds, a scale-free disk can not support modes with $\omega \neq 0$. The limiting case $\omega=0$ signals the onset of bifurcation or marginal stability of a particular mode. The most general form of $h_{(a)(b)}$ may be written as

$$
h_{(a)(b)}=\left(\begin{array}{llll}
h_{00} & h_{01} & h_{02} & h_{03} \\
h_{01} & h_{11} & h_{12} & h_{13} \\
h_{02} & h_{12} & h_{22} & h_{23} \\
h_{03} & h_{13} & h_{23} & h_{33}
\end{array}\right) e^{i m \phi},
$$

where the $10 h$ entries are functions of $\theta$ only. Geometrically, the metric coefficients are the inner products of the basis vectors

$$
g_{\mu \nu}=\frac{\partial}{\partial x^{\mu}} \cdot \frac{\partial}{\partial x^{\nu}}
$$

Since we are only considering polar perturbation, the system is symmetric about the equator and the metric is invariant under the diffeomorphism $\theta \rightarrow 2 \theta_{0}-\theta$. This implies the boundary condition

$$
h_{t \theta}=h_{\phi \theta}=h_{r \theta}=0 \Rightarrow h_{\mu 3}=0 \text { for } \mu \neq 3
$$

on the disk.

We proceed as follows. Project the left-hand side of (3-2) onto the LNRO, and write the result as $l_{(a)(b)}(1+n)^{2} e^{i m \phi} e^{N-Z} / r^{2}$. Expand $l_{(a)(b)}$ and replace the second derivatives of zeroth-order metric coefficients with the unperturbed Einstein equations (2-4). This will introduce singular terms on the disk. Since we require the metric to be continuous across the disk, all singular terms must balance for the first-order equations in $l_{(a)(b)}$, which are $l_{(a) 3}$ (the second-order equations are acceptable since the first derivatives will in general have a jump there). Miraculously, with the condition (3-3), all first-order equations are regular.

\footnotetext{
${ }^{2}$ This is not entirely unfamiliar. Recall that in the super-Hamiltonian formalism, the conjugate momentum to $x^{\mu}$ is $p_{\mu}$, which is what we vary, not $p^{\mu}$.
} 
To proceed further, we need to choose a gauge. Consider an infinitesimal coordinate transformation $x^{\mu} \rightarrow y^{\mu}=x^{\mu}+\xi^{\mu}(x)$, where $\xi^{\mu}$ is of the same magnitude as $h_{\mu \nu}$. This induces a transformation on the metric in the usual way,

$$
\begin{aligned}
g_{\alpha \beta}(x) & =\frac{\partial y^{\mu}}{\partial x^{\alpha}} \frac{\partial y^{\nu}}{\partial x^{\beta}} g_{\mu \nu}^{\prime}(x+\xi)=\left(\delta_{\alpha}^{\mu}+\xi_{, \alpha}^{\mu}\right)\left(\delta_{\beta}^{\nu}+\xi_{, \beta}^{\nu}\right)\left(g_{\mu \nu}^{\prime}+\xi^{\rho} g_{\mu \nu, \rho}^{\prime}\right) \\
& =g_{\alpha \beta}^{\prime}+g_{\mu \alpha}^{\prime} \xi_{, \beta}^{\mu}+g_{\mu \beta}^{\prime} \xi_{, \alpha}^{\mu}+\xi^{\rho} g_{\alpha \beta, \rho}^{\prime}=g_{\alpha \beta}^{\prime}+\left(£_{\xi} \mathbf{g}^{\prime}\right)_{\alpha \beta}=g_{\alpha \beta}^{\prime}+2 \xi_{(\alpha ; \beta)} .
\end{aligned}
$$

Thus, the coordinate freedom we have in general relativity corresponds to the gauge freedom $h_{\mu \nu} \rightarrow h_{\mu \nu}+2 \xi_{(\mu ; \nu)}$. As suggested by the boundary condition on the disk, we shall promote (3-3) to a gauge condition. There is one more degree of freedom which we will fix here. The total gauge thus reads

$$
h_{03}=h_{13}=h_{23}=0, \quad h_{11}=h_{33} .
$$

The last condition resembles the Regge-Wheeler gauge in spherical symmetry. With the gauge condition, we may write

$$
\begin{aligned}
h_{t t} & =r^{2 n} e^{N}\left[a+Q^{2} b-2 Q d\right] e^{i m \phi}, \quad h_{t \phi}=r^{n+1} e^{P}(d-Q b) e^{i m \phi}, \\
h_{t r} & =i r^{n} e^{Z / 2}(f-Q j) e^{i m \phi}, \quad h_{\phi \phi}=r^{2} e^{2 P-N} b e^{i m \phi}=e^{2 P-Z} h_{\theta \theta}, \\
h_{\phi r} & =i r e^{P+Z / 2-N} j e^{i m \phi}, \quad h_{r r}=e^{Z-N} c e^{i m \phi},
\end{aligned}
$$

which corresponds to

$$
h_{(a)(b)}=e^{i m \phi}\left(\begin{array}{cccc}
a & d & \text { if } & 0 \\
d & b & i j & 0 \\
i f & i j & c & 0 \\
0 & 0 & 0 & b
\end{array}\right) .
$$

The left-hand side of (3-2) now reads

$$
\begin{aligned}
l_{00} & =-a^{\prime \prime}-\left(\frac{1}{2} N^{\prime}+P^{\prime}\right) a^{\prime}+\frac{1}{2} N^{\prime} c^{\prime}+2 Q F d^{\prime} \\
& +\left\{-Q^{2}\left(\frac{1-n}{1+n}\right)^{2}-Q^{2} F^{2}-\tilde{\varepsilon} \Delta \frac{1+2 \gamma+v^{2}}{1-v^{2}}+e^{Z-2 P} \frac{m^{2}}{(1+n)^{2}}\right\} a \\
& +\left\{-2 Q^{2}\left(\frac{1-n}{1+n}\right)^{2}-Q^{2} F^{2}+\frac{2 n}{1+n}+2 e^{Z-2 P} Q^{2} \frac{m^{2}}{(1+n)^{2}}-\frac{1+2 \gamma+v^{2}}{1-v^{2}} \tilde{\varepsilon} \Delta\right\} b \\
& +\left\{Q^{2}\left(\frac{1-n}{1+n}\right)^{2}-\frac{2 n}{1+n}+Q^{2} e^{Z-2 P} \frac{m^{2}}{(1+n)^{2}}\right\} c \\
& +2\left\{Q\left[\left(\frac{1-n}{1+n}\right)^{2}-e^{Z-2 P} \frac{m^{2}}{(1+n)^{2}}+\left(N^{\prime}-P^{\prime}\right) F\right]-\frac{2 v(1+\gamma)}{1-v^{2}} \tilde{\varepsilon} \Delta\right\} d
\end{aligned}
$$




$$
\begin{aligned}
& -e^{Z / 2-P} \frac{2 Q m(1+2 n)}{(1+n)^{2}} f+e^{Z / 2-P} \frac{2 m\left(n+n Q^{2}-Q^{2}\right)}{(1+n)^{2}} j, \\
& l_{11}=-b^{\prime \prime}+\left(P^{\prime}-\frac{1}{2} N^{\prime}\right) a^{\prime}-P^{\prime} b^{\prime}+\left(\frac{1}{2} N^{\prime}-P^{\prime}\right) c^{\prime}+2 Q F d^{\prime} \\
& +\left\{-e^{Z-2 P} \frac{m^{2}}{(1+n)^{2}}-Q^{2}\left[\left(\frac{1-n}{1+n}\right)^{2}+F^{2}\right]\right\} a \\
& +\left\{e^{Z-2 P} \frac{\left(1-Q^{2}\right) m^{2}}{(1+n)^{2}}-Q^{2}\left[2\left(\frac{1-n}{1+n}\right)^{2}+F^{2}\right]-\frac{2}{1+n}\right\} b \\
& +\left\{e^{Z-2 P} \frac{m^{2}}{(1+n)^{2}}+Q^{2}\left(\frac{1-n}{1+n}\right)^{2}+\frac{2}{1+n}\right\} c \\
& +2 Q\left\{e^{Z-2 P} \frac{m^{2}}{(1+n)^{2}}+\left(\frac{1-n}{1+n}\right)^{2}+\left(N^{\prime}-P^{\prime}\right) F\right\} d \\
& +2 Q e^{Z / 2-P} \frac{m}{(1+n)^{2}} f-2 e^{Z / 2-P} \frac{m}{(1+n)^{2}}\left[Q^{2}(1-n)+n+2\right] j, \\
& l_{22}=-c^{\prime \prime}+\frac{1}{2}\left(Z^{\prime}-N^{\prime}\right) a^{\prime}+\frac{1}{2}\left(N^{\prime}-Z^{\prime}-2 P^{\prime}\right) c^{\prime}+Q^{2}\left(\frac{1-n}{1+n}\right)^{2} a \\
& +\left\{2 Q^{2}\left(\frac{1-n}{1+n}\right)^{2}+\frac{2 n(1-n)}{(1+n)^{2}}-\tilde{\varepsilon} \Delta\right\} b-2 Q\left(\frac{1-n}{1+n}\right)^{2} d \\
& +\left\{\left(1-Q^{2}\right) e^{Z-2 P} \frac{m^{2}}{(1+n)^{2}}+\tilde{\varepsilon} \Delta-Q^{2}\left(\frac{1-n}{1+n}\right)^{2}-\frac{2 n(1-n)}{(1+n)^{2}}\right\} c \\
& +Q e^{Z / 2-P} \frac{2 m n}{(1+n)^{2}} f+e^{Z / 2-P} \frac{2 m}{(1+n)^{2}}\left(Q^{2}-1-n Q^{2}\right) j, \\
& l_{01}=-d^{\prime \prime}+\frac{1}{2} Q F a^{\prime}+Q F b^{\prime}+\frac{1}{2} Q F c^{\prime}-P^{\prime} d^{\prime} \\
& +Q\left\{e^{Z-2 P} \frac{m^{2}}{(1+n)^{2}}-2 \frac{1-n}{(1+n)^{2}}\right\} b+Q\left\{e^{Z-2 P} \frac{m^{2}}{(1+n)^{2}}+2 \frac{1-n}{(1+n)^{2}}\right\} c \\
& +\left\{\left(1-Q^{2}\right)\left(\frac{1-n}{1+n}\right)^{2}-\tilde{\varepsilon} \Delta \gamma+\left(N^{\prime}-P^{\prime}\right)^{2}-Q^{2} F^{2}\right\} d \\
& -e^{Z / 2-P} \frac{2 m n}{(1+n)^{2}} f+2 Q e^{Z / 2-P} \frac{m(n-2)}{(1+n)^{2}} j, \\
& i l_{02}=f^{\prime \prime}+P^{\prime} f^{\prime}-Q F j^{\prime}+\left\{Q e^{Z-2 P} \frac{m^{2}}{(1+n)^{2}}+\left(P^{\prime}-\frac{1}{2} Z^{\prime}\right) Q F+2 \frac{(1+\gamma) v}{1-v^{2}} \tilde{\varepsilon} \Delta\right\} j \\
& +e^{Z / 2-P} \frac{m}{(1+n)^{2}}\left\{\frac{1}{2} Q(1-n) a+Q(1-n) b-\frac{1}{2}(n+3) Q c-(1-n) d\right\}
\end{aligned}
$$




$$
\begin{aligned}
& +\left\{-e^{Z-2 P} \frac{m^{2}}{(1+n)^{2}}-\left(N^{\prime}-\frac{1}{2} Z^{\prime}\right)^{2}+\frac{2 n}{(1+n)^{2}}+\frac{1}{2} Q^{2} F^{2}+\frac{v^{2}+\gamma}{1-v^{2}} \tilde{\varepsilon} \Delta\right\} f, \\
i l_{12} & =j^{\prime \prime}-Q F f^{\prime}+P^{\prime} j^{\prime}+Q\left\{-e^{Z-2 P} \frac{m^{2}}{(1+n)^{2}}-2 \frac{1-n}{(1+n)^{2}}+\left(\frac{1}{2} Z^{\prime}-N^{\prime}\right) F\right\} f \\
& +e^{Z / 2-P} \frac{m}{(1+n)^{2}}\left\{(1-n)\left(1+\frac{1}{2} Q^{2}\right) a+2(1-n) Q^{2} b\right. \\
& \left.-\left[1+n+\frac{1}{2} Q^{2}(1-n)\right] c-3(1-n) Q d\right\} \\
& +\left\{e^{Z-2 P} Q^{2} \frac{m^{2}}{(1+n)^{2}}+Q^{2}\left(\frac{1-n}{1+n}\right)^{2}+\frac{1+2 n-n^{2}}{(1+n)^{2}}\right. \\
& \left.-\left(P^{\prime}-Z^{\prime} / 2\right)^{2}+\frac{1}{2} Q^{2} F^{2}-\frac{1+\gamma v^{2}}{1-v^{2}} \tilde{\varepsilon} \Delta\right\} j .
\end{aligned}
$$

\subsection{Matter Content}

Recall that the disk is made of a two-dimensional perfect fluid. Explicitly, if we choose the equation of state $p=\gamma \varepsilon$, the unperturbed stress-energy tensor may be written as

$$
T^{\mu \nu}=\varepsilon\left[(1+\gamma) u^{\mu} u^{\nu}+\gamma g^{\mu \nu}\right], \text { for } \mu, \nu=t, \phi, r, \text { and } T_{\lambda \theta}=0 .
$$

In the presence of a perturbation, we still need to impose the condition that momentum flux and stress in the vertical direction vanish. Hence the first-order change in the stress-energy tensor is only for the upper-left $3 \times 3$ block:

$$
\delta T^{\mu \nu}=\delta \varepsilon\left[(1+\gamma) u^{\mu} u^{\nu}+\gamma g^{\mu \nu}\right]+\varepsilon\left[(1+\gamma)\left(\delta u^{\mu} u^{\nu}+u^{\mu} \delta u^{\nu}\right)-\gamma h^{\mu \nu}\right] .
$$

As usual, the four-velocity is normalized,

$$
\left(u^{\mu}+\delta u^{\mu}\right)\left(u^{\nu}+\delta u^{\nu}\right)\left(g_{\mu \nu}+h_{\mu \nu}\right)=-1 \Rightarrow \delta u^{\mu} u_{\mu}=-\frac{1}{2} u^{\mu} u^{\nu} h_{\mu \nu} .
$$

Projecting onto the LNRO frame, we have

$$
\delta T_{(a)(b)}=\delta \varepsilon\left[(1+\gamma) u_{(a)} u_{(b)}+\gamma \eta_{(a)(b)}\right]+\varepsilon\left[(1+\gamma)\left(\delta u_{(a)} u_{(b)}+u_{(a)} \delta u_{(b)}\right)-2 \gamma w_{(a)(b)}\right]
$$

and

$$
\delta u^{(a)} u_{(a)}=-u^{(a)} u^{(b)} w_{(a)(b)}
$$

where

$$
u^{(a)}=\left(\frac{1}{\sqrt{1-v^{2}}}, \frac{v}{\sqrt{1-v^{2}}}, 0,0\right), \quad \delta u^{(a)}=\left(\frac{x e^{i m \phi}}{\sqrt{1-v^{2}}}, \frac{y e^{i m \phi}}{\sqrt{1-v^{2}}}, \frac{i z e^{i m \phi}}{\sqrt{1-v^{2}}}, 0\right) .
$$


With this parameterization, the normalization condition reads

$$
x=(y+d) v+\frac{1}{2}\left(a+v^{2} b\right) .
$$

Next, we work out the equation of motion (EOM) for the perturbed quantities. Although it is not needed if we solve all six unknown metric perturbations directly, the EOM provides a consistency check for the algebraic mess that is notorious in general relativity. Furthermore, as we will see later, for the self-similar disk, the EOMs are all algebraic, which are much easier to handle than the full Einstein equations. A direct variation of $T_{; \nu}^{\mu \nu}=0$ reads

$$
\delta T_{; \nu}^{\mu \nu}+\delta \Gamma_{\rho \nu}^{\mu} T^{\rho \nu}+\delta \Gamma_{\rho \nu}^{\nu} T^{\rho \mu}=0,
$$

where

$$
\delta \Gamma_{\nu \rho}^{\mu}=\frac{1}{2} g^{\mu \alpha}\left(h_{\alpha \nu ; \rho}+h_{\alpha \rho ; \nu}-h_{\rho \nu ; \alpha}\right) .
$$

Just as in the unperturbed EOM, the $\mu=\theta$ component needs to be satisfied identically. Physically, this component gives the fluid evolution in the $\hat{\theta}$ direction, which is trivial in this case. If we assume that the metric coefficients are even about the equatorial plane, then the first derivatives are odd, and vanish upon integrating through the plane. The resulting equation only contains $h_{\nu \theta}$ where $\nu \neq 3$. This is another reason why the full gauge has to satisfy the boundary condition (3-3). Next, we consider the density perturbations. The zeroth-order density that is self-similar may be written as

$$
\varepsilon=\frac{A}{r^{2}} \delta\left(\theta-\theta_{0}\right)
$$

where $A=\tilde{\varepsilon} e^{N_{0}-Z_{0}} / 8 \pi$ is some constant. Conventionally, the equatorial plane is located at $\theta=\pi / 2$ in spherical polar coordinates. However, to reduce eigenvalues of the problem, we rescaled $\theta$ so that the disk is located at $\theta_{0}$. When the geometry is perturbed, this "hidden" eigenvalue will in general change, hence we need to vary $\theta_{0}$ as well. Thus, we obtain

$$
\begin{aligned}
\delta \varepsilon & =\frac{\delta A e^{i m \phi}}{r^{2}} \delta\left(\theta-\theta_{0}\right)+\frac{A}{r^{2}}\left[\delta\left(\theta-\theta_{0}-\theta_{1} e^{i m \phi}\right)-\delta\left(\theta-\theta_{0}\right)\right] \\
& =\frac{e^{i m \phi}}{r^{2}}\left[\delta A \delta\left(\theta-\theta_{0}\right)-A \delta^{\prime}\left(\theta-\theta_{0}\right) \theta_{1}\right]
\end{aligned}
$$


Projecting the right-hand side of (3-2) onto the LNRO and writing the result as $H_{(a)(b)}(1+$ $n)^{2} e^{i m \phi} e^{N-Z} / r^{2}$, the nonzero components are ${ }^{3}$

$$
\begin{aligned}
H_{00} & =\frac{1+2 \gamma+v^{2}}{1-v^{2}} \zeta \Delta+\left[-\frac{1+\gamma v^{2}+Q v(1+\gamma)}{1-v^{2}} a+\frac{v(2 v-Q)(1+\gamma)}{1-v^{2}} b\right. \\
& \left.+\frac{(v-Q)(1+\gamma)-Q v^{2}(1+\gamma)}{1-v^{2}} d+4 v \frac{1+\gamma}{1-v^{2}} y\right] \tilde{\varepsilon} \Delta \\
H_{01} & =-\frac{2 v(1+\gamma)}{1-v^{2}} \zeta \Delta+\left[-v \frac{\left(2+v^{2}\right)(1+\gamma)}{1-v^{2}} b-\frac{2\left(1+\gamma v^{2}\right)+\gamma+v^{2}}{1-v^{2}} d\right. \\
& \left.-2 \frac{(1+\gamma)\left(1+v^{2}\right)}{1-v^{2}} y\right] \tilde{\varepsilon} \Delta, \\
i H_{02} & =\tilde{\varepsilon} \Delta\left[\frac{\gamma+\gamma v^{2}+2}{1-v^{2}} f+2 v \frac{1+\gamma}{1-v^{2}} j+2 \frac{1+\gamma}{1-v^{2}} z\right] \\
H_{11} & =\frac{1+v^{2}+2 \gamma v^{2}}{1-v^{2}} \zeta \Delta+\left[\frac{\gamma+3 v^{2}+2 \gamma v^{2}}{1-v^{2}} b+3 v \frac{1+\gamma}{1-v^{2}} d+4 v \frac{1+\gamma}{1-v^{2}} y\right] \tilde{\varepsilon} \Delta, \\
i H_{12} & =-\tilde{\varepsilon} \Delta\left[2 v \frac{1+\gamma}{1-v^{2}} f+\frac{\gamma+\gamma v^{2}+2 v^{2}}{1-v^{2}} j+2 v \frac{1+\gamma}{1-v^{2}} z\right] \\
H_{22} & =\zeta \Delta+\gamma c \tilde{\varepsilon} \Delta,
\end{aligned}
$$

where

$$
\zeta \Delta=8 \pi \delta \varepsilon \frac{r^{2} e^{Z-N}}{1+n} e^{-i m \phi} .
$$

If we use the definition of $\delta \varepsilon$, the above expression simplifies to

$$
\zeta \Delta=\tilde{\varepsilon}_{1} \Delta-\tilde{\varepsilon} e^{N_{0}-Z_{0}} e^{Z-N} \Delta^{\prime} \Theta_{1}=\tilde{\varepsilon}_{1} \Delta+\tilde{\varepsilon}\left(Z_{0}^{\prime}-N_{0}^{\prime}\right) \Theta_{1} \Delta \Rightarrow \zeta=\tilde{\varepsilon}_{1}+\tilde{\varepsilon}\left(Z_{0}^{\prime}-N_{0}^{\prime}\right) \Theta_{1},
$$

where we integrated by parts on the last term. In fact, the explicit form of $\zeta$ is not required here since $\tilde{\varepsilon}_{1}$ and $\Theta_{1}$ never appear independently in the equations. This observation suggests that $\Theta_{1}$ is a second order effect.

\subsection{Boundary Conditions}

¿From symmetry, all the non-axisymmetric metric components should vanish on the axis (where $\Theta=0$ ). Thus we can impose the conditions

$$
a=b=c=d=f=j=0 .
$$

\footnotetext{
${ }^{3}$ Actually $H_{33}$ is nonzero too, but it contains exactly the singular terms from $l_{33}$.
} 
On the disk, the delta functions give rise to a jump in the derivatives of the perturbation functions using the second-order equations. Integrating across the disk, we have

$$
\begin{aligned}
2 a^{\prime} & =\tilde{\varepsilon}\left\{\frac{v^{2}+2 \gamma-\gamma v^{2}-Q v(1+\gamma)}{1-v^{2}} a+\frac{1+2 \gamma+3 v^{2}+2 \gamma v^{2}-Q v(1+\gamma)}{1-v^{2}} b\right. \\
& \left.+\frac{(1+\gamma)\left(5 v-Q-Q v^{2}\right)}{1-v^{2}} d+\frac{4 v(1+\gamma)}{1-v^{2}} y\right\}+\frac{1+2 \gamma+v^{2}}{1-v^{2}} \zeta, \\
2 b^{\prime} & =\tilde{\varepsilon}\left\{\frac{2 \gamma v^{2}+3 v^{2}+\gamma}{1-v^{2}} b+\frac{v(1+\gamma)}{1-v^{2}}(3 d+4 y)\right\}+\frac{1+v^{2}+2 \gamma v^{2}}{1-v^{2}} \zeta, \\
2 c^{\prime} & =\tilde{\varepsilon}\{b-(1-\gamma) c\}+\zeta, \\
2 d^{\prime} & =\tilde{\varepsilon}\left\{-\frac{v\left(v^{2}+2\right)(1+\gamma)}{1-v^{2}} b-\frac{3 \gamma v^{2}+2+v^{2}}{1-v^{2}} d-\frac{2\left(1+v^{2}\right)(1+\gamma)}{1-v^{2}} y\right\} \\
& -\frac{2 v(1+\gamma)}{1-v^{2} \zeta,} \\
-2 f^{\prime} & =\tilde{\varepsilon}\left\{\frac{2+\gamma v^{2}-v^{2}}{1-v^{2}} f+\frac{2(1+\gamma)}{1-v^{2}} z\right\}, \\
-2 j^{\prime} & =\tilde{\varepsilon}\left\{-\frac{2 v(1+\gamma)}{1-v^{2}} f+\frac{1-\gamma-2 v^{2}}{1-v^{2}} j-\frac{2 v(1+\gamma)}{1-v^{2}} z\right\} .
\end{aligned}
$$

The equations of motion in the disk read

$$
\begin{aligned}
& 2 v a-\left[v\left(v^{2}+2\right)+Q \frac{4 \gamma v^{2}+\gamma+2+3 v^{2}}{1+\gamma}\right] b-(v+Q) c-4 Q v d \\
& -2\left(1+v^{2}+2 Q v\right) y-\frac{2}{m} e^{P-Z / 2}[2 n-Q v(1-n)] z-2\left[Q \frac{1+\gamma v^{2}}{1+\gamma}+v\right] \frac{\zeta}{\tilde{\varepsilon}}=0, \\
& \left(1+v^{2}\right) a-\left\{\frac{\gamma\left(1-v^{2}\right)}{1+\gamma}+4 Q v+Q v^{3}+3 v^{2}\right\} b-(v+Q) v c-2\left(1+v^{2}\right) Q d \\
& -2\left(Q+Q v^{2}+2 v\right) y-\frac{2 v}{m} e^{P-Z / 2}(1+n) z-2\left\{Q v+\frac{v^{2}+\gamma}{1+\gamma}\right\} \frac{\zeta}{\tilde{\varepsilon}}=0 \\
& v Q a+\left(2 Q+Q v^{2}+2 v\right) v b+2\left(Q+Q v^{2}+v\right) d+2 m \frac{Q+v}{1-n} e^{Z / 2-P} f \\
& +2\left(Q+Q v^{2}+2 v\right) y+2 v m \frac{Q+v}{1-n} e^{Z / 2-P} j+2 m \frac{Q+v}{1-n} e^{Z / 2-P} z=0 .
\end{aligned}
$$

Equations (3-12), (3-13) and (3-14) form the complete set of boundary conditions.

\section{Newtonian Limit}

As shown in Shu et. al. (2000), the Newtonian magnetized isothermal singular disks allow bifurcation to non-axisymmetric equilibria if the rotation velocity is high enough. 
Specifically, the onset of bifurcation occurs at

$$
D^{2}=\frac{m}{m+2}, \text { for } m \geq 2
$$

where $D$ is the ratio of rotation speed to magnetosonic speed (which equals the sound speed at zero magnetization). Our equation should yield the same result in the limit $v \ll 1$ and $\gamma \ll 1$. As usual, the Newtonian limit is recovered by setting

$$
g_{00}=-1-2 \Phi+O\left(v^{4}\right), \quad g_{0 j}=O\left(v^{3}\right), \quad g_{i j}=\eta_{i j}+O\left(v^{2}\right),
$$

where $\Phi$ is the Newtonian gravitational potential and is of order $v^{2}$. We start with the unperturbed axisymmetric solution described by the metric (2-1) and the associated Einstein equations (2-4) and (2-5). The Newtonian limit becomes

$$
\begin{aligned}
& \Phi=(N / 2+n \log r)\left(1-Q^{2}\right)=O\left(v^{2}\right)+O\left(v^{4}\right), \\
& e^{2 P-N}=\sin ^{2} \theta+O\left(v^{2}\right), \quad e^{P} Q=O\left(v^{3}\right), \quad Z-N=O\left(v^{2}\right),
\end{aligned}
$$

or

$$
N \sim n \sim Z \sim \Theta_{0}-\frac{\pi}{2} \sim e^{P}-\sin \theta \sim \gamma \sim v^{2}, \text { and } Q \sim v^{3} .
$$

The equation for $N$ reads

$$
N^{\prime \prime}+N^{\prime} \cot \Theta+2 n=\tilde{\varepsilon} \Delta, \quad N^{\prime}(0)=0,
$$

which has the solution

$$
N^{\prime}=-2 n \tan \frac{\Theta}{2}, \quad 4 n \tan \frac{\Theta_{0}}{2}=\tilde{\varepsilon} .
$$

Since $\Theta_{0} \approx \frac{\pi}{2}$, we have $\tilde{\varepsilon} \approx 4 n$, which means it's also small. Thus, the equation for $P$ becomes

$$
0=2 \cot \Theta_{0} \Rightarrow \Theta_{0} \equiv \frac{\pi}{2}, \text { and } \Theta \equiv \theta .
$$

$Z$ may be most directly computed through the second constraint equation relating it to $N^{\prime}$,

$$
Z^{\prime}=2 n N^{\prime}=-4 n^{2} \tan \frac{\theta}{2}=O\left(v^{4}\right) .
$$

Finally, the last constraint equation may be solved order by order. The $O\left(v^{4}\right)$ terms are identically zero by our solution of $N^{\prime}$. The next order is $O\left(v^{6}\right)$, which reads

$$
\begin{aligned}
& \left(Q \cot \theta-Q^{\prime}\right)^{2}-Q^{2}-2 Q \cot \theta\left(Q \cot \theta-Q^{\prime}\right)=0 \\
\Rightarrow & \log Q^{\prime}=\csc \theta \\
\Rightarrow & Q=C \tan \frac{\theta}{2} .
\end{aligned}
$$


Integrating the dynamic equation for $\mathrm{Q}$, we obtain a jump condition, which in this limit reads

$$
C=2 n(C+2 v) \Rightarrow C=4 n v .
$$

Putting everything together, the limiting solution is

$$
\begin{aligned}
& n=v^{2}+\gamma, \quad \tilde{\varepsilon}=4 n, \quad P=\log \sin \theta \\
& N^{\prime}=-2 n \cot \frac{\theta}{2}, \quad Q=4 n v \tan \frac{\theta}{2}, \quad Z^{\prime}=-4 n^{2} \tan \frac{\theta}{2} .
\end{aligned}
$$

Of course, in the purely Newtonian case, $Q$ and $Z$ are taken to be 0 since they are of higher order. A simple integration yields

$$
N=-2 n \log (1-\cos \theta) / 2 \Rightarrow \Phi=-\left(v^{2}+\gamma\right) \log \left[\frac{r}{2}(1-\cos \theta)\right]
$$

which is the correct result for a hot Mestel disk.

In the presence of a perturbation, we still demand the Newtonian limit to be valid. Thus, the only nontrivial metric perturbation is in $g_{00}$ which is $a$, and

$$
g_{00}=-r^{2 n} e^{N}\left(1-a e^{i m \phi}\right)
$$

Expanding everything to leading order in $v$, the Einstein equations reduce to

$$
-a^{\prime \prime}-\cot \theta a^{\prime}+\frac{m^{2}}{\sin ^{2} \theta} a=\zeta \Delta .
$$

This is nothing more than the Poisson equation for the perturbed potential $V=-a / 2$.

Next, we'll derive the Newtonian version of the equations of motion. From the Poisson equation, we know that $a$ is of order $\zeta$, which is in turn of order $v^{2}$, while $y$ and $z$ are both of order $v$. It is worthwhile to point out that we are doing a two-parameter expansion, one in $v$ and one in the perturbation. In this limit, the EOM becomes

$$
\begin{aligned}
& y+v \frac{\zeta}{\tilde{\varepsilon}}=0, \\
& \frac{a_{0}}{2}-2 v y-\frac{v}{m} z-\left(v^{2}+\gamma\right) \frac{\zeta}{\tilde{\varepsilon}}=0, \\
& 2 y+m z=0
\end{aligned}
$$

where $a_{0}$ is evaluated on the disk, of course. A not so trivial calculation by Galli et. al. (2001) shows that $a_{0}=\zeta / 2 m$. Combining all three equations, we have (recall $\tilde{\varepsilon}=4\left(v^{2}+\gamma\right)$ )

$$
\frac{v^{2}+\gamma}{m}+v^{2}-\frac{2 v^{2}}{m^{2}}-\gamma=0 \Rightarrow \frac{v^{2}}{\gamma}=\frac{m}{m+2} \text { or } m=1 \text {. }
$$


This is the Newtonian bifurcation point obtained by Shu et. al. (2000). ${ }^{4}$

\section{Numerical Implementation}

Equation (3-2) is linear in the metric perturbations. Therefore it is ideal for finite differencing, which transforms the differential equations into a matrix equation. Consider a vector

$$
\mathbf{V}=\mathbf{a} \oplus \mathbf{b} \oplus \mathbf{c} \oplus \mathbf{d} \oplus \mathbf{f} \oplus \mathbf{j} \oplus \mathbf{x}
$$

where

$$
\mathbf{a}=\left(a_{1}, a_{2}, \ldots a_{N}\right), \quad a_{i}=a\left(\Theta_{i}\right), \quad \Theta_{N}=\Theta_{\max }
$$

etc., and

$$
\mathbf{x}=\left(\tilde{\varepsilon}_{1}, y, i z\right)
$$

We do not include the values of the perturbation on the pole, since they all vanish by the boundary conditions (3-12). For simplicity, let's use a uniform grid as we did in the unperturbed solution. Thus

$$
\Theta_{i}=i h, \quad h=\Theta_{\max } / N .
$$

With these definitions, the differential operators may be written as

$$
a_{i}^{\prime}=\frac{a_{i+1}-a_{i-1}}{2 h}, \quad a_{i}^{\prime \prime}=\frac{a_{i+1}-2 a_{i}+a_{i-1}}{h^{2}} .
$$

It is easy to check that these expressions are accurate to second order. On the boundary, the situation is a little bit trickier. To find the correct differencing scheme, let's expand the function on the axis to second order:

$$
\begin{aligned}
& a_{1}=a_{0}+h a_{0}^{\prime}+\frac{1}{2} h^{2} a_{0}^{\prime \prime}, \\
& a_{2}=a_{0}+2 h a_{0}^{\prime}+2 h^{2} a_{0}^{\prime \prime} .
\end{aligned}
$$

Taking the proper linear combinations, we have

$$
\begin{aligned}
& a_{0}^{\prime}=\frac{4 a_{1}-3 a_{0}-a_{2}}{2 h}, \\
& a_{0}^{\prime \prime}=\frac{a_{2}-2 a_{1}+a_{0}}{h^{2}} .
\end{aligned}
$$

\footnotetext{
${ }^{4}$ The conclusion that eccentric $m=1$ bifurcations can occur at any level of disk rotation is flawed, as are the analyses of Syer \& Tremaine (1996); Shu et. al. (2000) and Galli et. al. (2001) on this point (Toomre, personal communication; Shu, Toomre, Tremaine, in preparation). Except for one special rotation rate (the true bifurcation value), the stress tensor is nonzero at the origin (implying a physically unrealistic steady flow of momentum from the origin to infinity), even though it has zero divergence.
} 
Similarly, on the disk,

$$
\begin{aligned}
& a_{N-1}=a_{N}-h a_{N}^{\prime}+\frac{1}{2} h^{2} a_{N}^{\prime \prime}, \\
& a_{N-2}=a_{N}-2 h a_{N}^{\prime}+2 h^{2} a_{N}^{\prime \prime} .
\end{aligned}
$$

Thus,

$$
\begin{aligned}
& a_{N}^{\prime}=\frac{3 a_{N}-4 a_{N-1}+a_{N-2}}{2 h}, \\
& a_{N}^{\prime \prime}=\frac{a_{N-2}-2 a_{N-1}+a_{N}}{h^{2}} .
\end{aligned}
$$

The set of equations (3-2) may now be written as a matrix equation

$$
\mathbf{M} \cdot V=0 .
$$

It is not too hard to convince oneself that the matrix $\mathbf{M}$ is $(6 N+3) \times(6 N+3)$. After finite differencing, each component of $l_{i j}$ is represented by a $(6 N+3) \times N$ submatrix, where the left-hand side is evaluated at $\Theta=h, 2 h, . .(N-1) h$. The last row in this submatrix is replaced by the boundary condition (3-13). We thus fill the first $6 N$ rows of $\mathbf{M}$. The very last three rows are the remaining boundary conditions of (3-12).

In order for (5-5) to have nontrivial solutions $V$, the determinant of $M$ must vanish. Schematically, the elements of $M$ are nonlinear functions of the azimuthal quantum number $m$, and unperturbed metric coefficients (which are, in turn, functions of $v$ and $\gamma$ ). Thus, for

a given value of $m$ and $\gamma$, the problem of stability analysis is reduced to root finding of the equation

$$
\left|M_{m, \gamma}(v)\right|=0
$$

\section{Results}

We scan the entire solution space looking for the solution to (5-6). As expected, the bifurcation points form two sets of tracks in the $\gamma-v^{2}$ space. The behaviors of these two tracks are fundamentally different, since they are caused by two different mechanisms. We will discuss them separately.

\subsection{Radiation Driven Neutral Modes}

The first set of tracks is shown in Fig 1. These modes are believed to be the analog of Rossby modes first discovered by Chandrasekhar in 1970 and subsequently studied extensively in the context of neutron stars. Even though our self-similar disk geometry is in some 
sense infinitely different from the finite spherical geometry of neutron stars, the underlying mechanism for these neutral modes can still be understood if one is comfortable with the idea of gravitational radiation with infinite wavelength. To make a bad situation even worse, since our disks are formally infinite in size, one is never able to reach the radiation zone to study the gravitational wave. However, since the original argument of Chandrasekhar did not rely crucially on the asymptotic flatness of spacetime, the qualitative result still holds in this pathological case. Consider a non-axisymmetric disturbance in the disk which moves at a velocity $v_{1}<v$. As a result, the total angular momentum (or more appropriately, the specific angular momentum) decreases. These perturbations will in general radiate due to non-axisymmetry. If in the LNRO $v_{1}<0$, then gravitational radiation carries away negative angular momentum, which damps the amplitude of perturbation, and these modes are stable. On the other hand, if $v_{1}>0$, gravitational radiation carries away positive angular momentum, and thus the amplitude of perturbation has to grow to make the total angular momentum more negative.

For a given equation of state specified by $\gamma$, the radiation-driven neutral modes occur at lower Mach number for increasing $m$. This is expected from the analysis of Friedman \& Schutz $(1975,1978 \mathrm{a}, \mathrm{b})$. In fact, the onset of instability for $m \rightarrow \infty$ occurs at zero rotational velocity. However, for a realistic system, the strength of a particular unstable mode is intimately related to the magnitude of the imaginary part of its frequency. For the $m \rightarrow \infty$ mode, even though it is formally unstable at zero rotation, the characteristic growth time scale is infinite. This is due to the fact that multipole radiation is exceedingly weak for higher values of $m$. If we truncate the self-similar disk, the strongest unstable modes - that is, the modes with shortest growth time - are still the ones with small $m$. In the absence of viscosity, these modes will grow until the non-linear effects set in and limit the final rotation speed of the full disk.

In addition to the R-mode tracks, the $Q=1$ curve is also plotted in Fig 1 . We would like to remind the readers that these tracks represent models where the characteristic frequency of a given mode becomes purely real, i.e., modes that are marginally stable. It is not too difficult to convince oneself that every mode in the background of an ergoregion is unstable. Using the simplistic picture of retrograde disturbances, we see that in the ergoregion, every mode has to propagate in the direction of the underlying disk as seen by an LNRO, and gravitational radiation will drive them unstable. Furthermore, as Friedman (1978) demonstrated, a spacetime with an ergoregion is unstable even under scalar and vector perturbations. From Fig 1 , the $m=2$ track is entirely above the $Q=1$ curve and so is part of the $m=3$ track. In the presence of perturbing matter fields other than those in the disk itself, the ergoregion is likely to put a more stringent limit on the maximum rotation rate for a disk. 
The question arises whether the growth of R-modes might be suppressed by viscous torques due to, e.g., a magneto-rotational instability (MRI) acting in the disk Balbus \& Hawley (1991). Since the viscous effects must act to erase non-axial symmetries of length scale $\sim r / m$, the damping time associated with linear global R-modes must be the diffusion time scale $t_{D} \sim r^{2} /\left(m^{2} \nu\right)$, where $\nu$ is the effective MRI kinematic viscosity. If relativistic disks are even weakly magnetized and electrically conducting, as their Newtonian counterparts are believed to be, for $m \sim 1$, the time scale $t_{D}$ may be only two orders of magnitude longer than the dynamical timescale $r / v$. R-mode spin-down for such disks may then be effectively suppressed by the MRI viscosity, but calculations of non-self-similar relativistic disks are needed to answer definitively whether the growth rate of R-modes (here zero) can overcome the viscous damping.

\subsection{Newtonian Bifurcation Track}

The second set is the generalization of Newtonian bifurcation computed by Shu et. al. (2000). In Fig 2, we plotted these extended "Newtonian" tracks for $m=2,3,4,5$, and $\infty$. The finite- $m$ values are the numerical result from solving (5-6). When $m$ becomes large, we may approximate it by a continuous variable and use it as a parameter in the asymptotic expansion. Assume all the perturbation amplitudes remain infinitesimal in the large- $m$ limit, then the coefficients of each power of $m$ need to vanish independently. This requirement translates to

$$
\begin{aligned}
& a+2 Q^{2} b+Q^{2} c-2 d=0, \quad Q(1+2 n) f=\left(n+n Q^{2}-Q^{2}\right) j \\
& -a+\left(1-Q^{2}\right) b+c+2 Q d=0, \quad Q f=\left[Q^{2}(1-n)+n+2\right] j, \\
& c=0, \quad n Q f=-\left(Q^{2}-1-n Q^{2}\right) j \\
& Q b+Q c=0, \quad n f=Q(n-2) j, \\
& \frac{1}{2} Q(1-n) a+Q(1-n) b-\frac{1}{2}(n+3) Q c-(1-n) d=0, \quad Q j=f, \quad Q f=Q^{2} j, \\
& (1-n)\left(1+\frac{1}{2} Q^{2}\right) a+2(1-n) Q^{2} b-\left[1+n+\frac{1}{2} Q^{2}(1-n)\right] c-3(1-n) Q d .
\end{aligned}
$$

Regardless of whether the disk is rotating, these (linear, homogeneous) equations only have trivial solutions. This is the familiar Cowling approximation where the metric perturbations vanish in the large- $m$ limit. These Cowling modes may be understood in the following schematic way. Recall that in the Newtonian limit, we can invert Poisson's equation via a Green's function, and obtain an integral representation of the gravitational field. This procedure can also be done for the full Einstein equations in principle, although not analytically. Mathematically, the integral representation of metric perturbations is effectively an 
average of the matter perturbation times the Green's function. Therefore, in the $m \rightarrow \infty$ limit, the gravitational field is indifferent to the matter perturbation, since it averages to the axisymmetric equilibrium over any finite angular integration. This line of argument may be made mathematically rigorous with some more thought, but it is not necessary here.

In the absence of a metric perturbation, the equations of motion now simplify to

$$
\left(1+v^{2}+2 Q v\right) y+\left[Q \frac{1+\gamma v^{2}}{1+\gamma}+v\right] \frac{\zeta}{\tilde{\varepsilon}}=0, \quad\left(Q+Q v^{2}+2 v\right) y+\left[Q v+\frac{v^{2}+\gamma}{1+\gamma}\right] \frac{\zeta}{\tilde{\varepsilon}}=0 .
$$

Eliminating the factor $\zeta / \tilde{\varepsilon} y$, we can combine these equations to give

$$
\left(1+v^{2}+2 Q v\right)\left(Q v+Q v \gamma+v^{2}+\gamma\right)=\left(Q+Q \gamma v^{2}+v+v \gamma\right)\left(Q+Q v^{2}+2 v\right)
$$

The last equation implicitly defines a surface of neutral modes $Q\left(\theta_{0}\right)=f(\gamma, v)$. Recall that the solution space of axisymmetric disks can also be viewed as a two-dimensional surface defined by $Q\left(\theta_{0}\right)=g(\gamma, v)$. Thus the intersection of these two surfaces gives the bifurcation curve in the $\gamma-v$ space. This curve is also plotted in Fig. 2.

Near the origin, these bifurcations tracks recover the Newtonian result, where the bifurcation point is located at

$$
\frac{v^{2}}{\gamma}=\frac{m}{m+2}, \quad v, \gamma \rightarrow 0
$$

As $\gamma$ increases, the relativistic effects become important. Intriguingly, the point of bifurcation occurs at lower Mach number for a relativistic disk than in its Newtonian counterpart. The curve defined by $Q=1$ on the disk is again plotted. As seen in Fig 2, these tracks are confined in the portion of solution space where the time-like Killing vector remains timelike. In fact, the only place where a bifurcating neutral mode is allowed in the ergoregion is for $m=\infty$ and $\gamma=1$. Even then, the spacetime is only marginally ergo-like in the sense that $Q \rightarrow 1^{-}$on the disk. To understand this phenomenon, we examine the velocity perturbation corresponding to the bifurcating neutral modes. In the Newtonian limit, we can evaluate it analytically [see equation (4-5), or equation (21) of Shu et. al. (2000)]

$$
\delta v=y e^{i m \phi}=-\frac{v}{4\left(v^{2}+\gamma\right)} \delta \tilde{\varepsilon}
$$

In general, this component of the eigenvector needs to be computed numerically. Actually, the exact form, or even the magnitude of this velocity perturbation, is not important. It suffices to know that $\delta v$ for this mode always has the opposite sign of $\delta \tilde{\varepsilon}$. In the linear perturbation regime, where we can still apply the superposition principle, this observation has the following physical picture. The full disk solution has two components. One is the axisymmetric equilibrium rotating at $v$ in the $+\hat{\phi}$ direction with energy density given by $\varepsilon \propto$ 
$\tilde{\varepsilon} \delta\left(\theta-\theta_{0}\right) / r^{2}$. The second component is the non-axisymmetric perturbation, which is a disk of infinitesimal energy density $\delta \tilde{\varepsilon}$, and infinitesimal velocity field $\delta u^{\mu}$. Along the bifurcation track, this perturbation disk is always counter-rotating. The empirical result of Fig 2 leads us to postulate that the existence of counter-rotating non-axisymmetric disturbance is another necessary condition for bifurcation, at least for the disk geometry. A corollary is that the full non-axisymmetric disk spacetime can not have a stable ergoregion. This conjecture leads naturally to the confinement of bifurcation tracks in the portion of the solution space without ergoregions. For models with $Q>1$ on the disk, the time-like Killing vector becomes space-like, and thus no counter-rotating trajectory is allowed.

Can we elevate this conjecture to apply to a more generic relativistic non-axisymmetric equilibrium? The answer is a cautious yes. Without digressing too much into the mathematical structure of Riemannian geometry, we would like to offer the following plausibility argument. Suppose we are able to construct a fully nonlinear, non-axisymmetric stationary solution to the Einstein field equations. It can not have an event horizon, since black holes can not have "hair" (in this case, "hair" refers to mass multipole moments). Furthermore, in the absence of a space-like Killing vector $\boldsymbol{\partial}_{\phi}$, the non-vanishing component of $g_{t j}$ will generate a time-dependent quadrupole moment as seen by an inertial observer. As a result, gravitational radiation will continue to carry away angular momentum and energy until the system is either axisymmetric or static. In this aspect, the relativistic non-axisymmetric equilibria are analogous to the Dedekind ellipsoids, where the figure axes are static in an inertial frame, and the configuration is supported by pressure and internal motion. It can be shown (see, e.g., Chandrasekhar 1983) that a static metric can always be brought to the diagonal form after appropriate coordinate transformations. With a diagonal metric, the ergoregion defined by $g_{t t}=0$ coincides with the event horizon. Therefore, the absence of an event horizon means a non-axisymmetric static solution does not have an ergoregion.

\section{Summary and Discussion}

We performed a linear stability analysis of the relativistic self-similar disk against nonaxisymmetric perturbations. For simplicity, we restricted the class of perturbation under consideration to be self-similar and polar. Mathematically, this means the scaling law is preserved and the metric is symmetric about the midplane even in the presence of perturbation.

As expected, the Newtonian bifurcations found by Shu et. al. (2000) and Galli et. al. (2001) have extensions into the fully relativistic regime. These tracks seem to exist only in models which do not admit an ergoregion. The corresponding velocity perturbation is strictly negative for any positive energy density increase. We thus hypothesize that in 
addition to the existence of neutral modes, retrograde disturbance may also be a necessary condition for bifurcation to non-axisymmetric disk equilibria. This line of arguments leads us to speculate that the non-axisymmetric equilibrium solutions in general can not have ergoregions. We have no proof that the behavior is generic, whereas the bifurcation of rapidly rotating axisymmetric equilibria to non-axisymmetric forms probably is.

In addition, we have discovered the onset of R-mode instability, which is driven by gravitational radiation. The marginal stability tracks follow the qualitative behavior first discussed by Friedman \& Schutz (1978b), and the result here is probably also generic. For a self-similar disk, the entire $m=2$ neutral-mode tracks and part of $m=3$ occur in models with ergocones. We believe that in general for a fixed equation of state, the onset of instability occurs either at the ergoregion formation, or the tracks we computed, whichever have lower velocity.

These studies are the first step to constructing fully non-axisymmetric relativistic equilibria. For a given value of $\gamma$, if the axisymmetric state contracts quasi-statically by shedding angular momentum, the linear rotational velocity will increase. This evolution represents a vertical line in Figs 1 and 2. Eventually, the velocity will reach a value where a nonaxisymmetric mode becomes unstable. If they undergo gravitational collapse, the secular instability will most likely survive over many dynamic time scales (which, in the purely selfsimilar case, is infinite). Therefore, the collapse will be fundamentally non-axisymmetric. For simplicity, we have only considered each Fourier component independently. In the linear perturbation regime, the effect of a general non-axisymmetric disturbance can always be decomposed into its Fourier components with each component decoupled from others. When the amplitudes become finite, our Fourier series will fail to converge, and a more detailed analysis is required.

The current work serves as a spring board to one of the ultimate challenges in numerical relativity - the fully nonlinear numerical simulation of a non-axisymmetric collapse. Only then can we answer questions such as whether the central singularities of objects like relativistic SIDs remain naked.

We thank the referee for valuable comments. This work has been supported by Academia Sinica through the Distinguished Postdoctoral Research Fellowship awarded to MJC, and by National Science Council through grants NSC 91-2112-M-007-29 and NSC 92-2112-M-001062 awarded to FHS. 


\section{REFERENCES}

Balbus, S. A. \& Hawley, J. F. 1991, ApJ, 376, 214.

Bardeen, J. M. \& Wagoner, R. V. 1971, ApJ, 167, 359.

Cai, M. J. \& Shu, F. H. 2002, ApJ, 567, 477.

Cai, M. J. \& Shu, F. H. 2003, ApJ, 583, 391.

Cai, M. J. \& Shu, F. H. 2004, in preparation.

Chandrasekhar, S. 1969, "Ellipsoidal Figures of Equilibrium", Yale University Press.

Chandrasekhar, S. 1970a, ApJ, 161, 561.

Chandrasekhar, S. 1970b, ApJ, 161, 571.

Chandrasekhar, S. 1983, "The Mathematical Theory of Black Holes", Oxford University Press.

Friedman, J. 1978, CmP, 63, 243.

Friedman, J. \& Schutz, B. 1975, ApJ, 200, 204.

Friedman, J. \& Schutz, B. 1978a, ApJ, 221, 937.

Friedman, J. \& Schutz, B. 1978b, ApJ, 222, 281.

Galli, D., Shu, F. H., Laughlin, G. \& Lizano, S. 2001, ApJ, 551, 367.

Hayashi, C., Narita, S., \& Miyama, S. M. 1982, PThPh, 68, 1949.

Mestel, L. 1963, MNRAS, 126, 533.

Lynden-Bell, D. \& Pineault, S. 1978, MNRAS, 185, 695.

Syer, D. \& Tremaine, S. 1996, MNRAS, 281, 925.

Shu, F. H., Laughlin, G., Lizano, S. \& Galli, D. 2000, ApJ, 525, 190.

Toomre, A. 1982, ApJ, 259, 535.

Wald, R. 1984, "General Relativity", University of Chicago Press. 


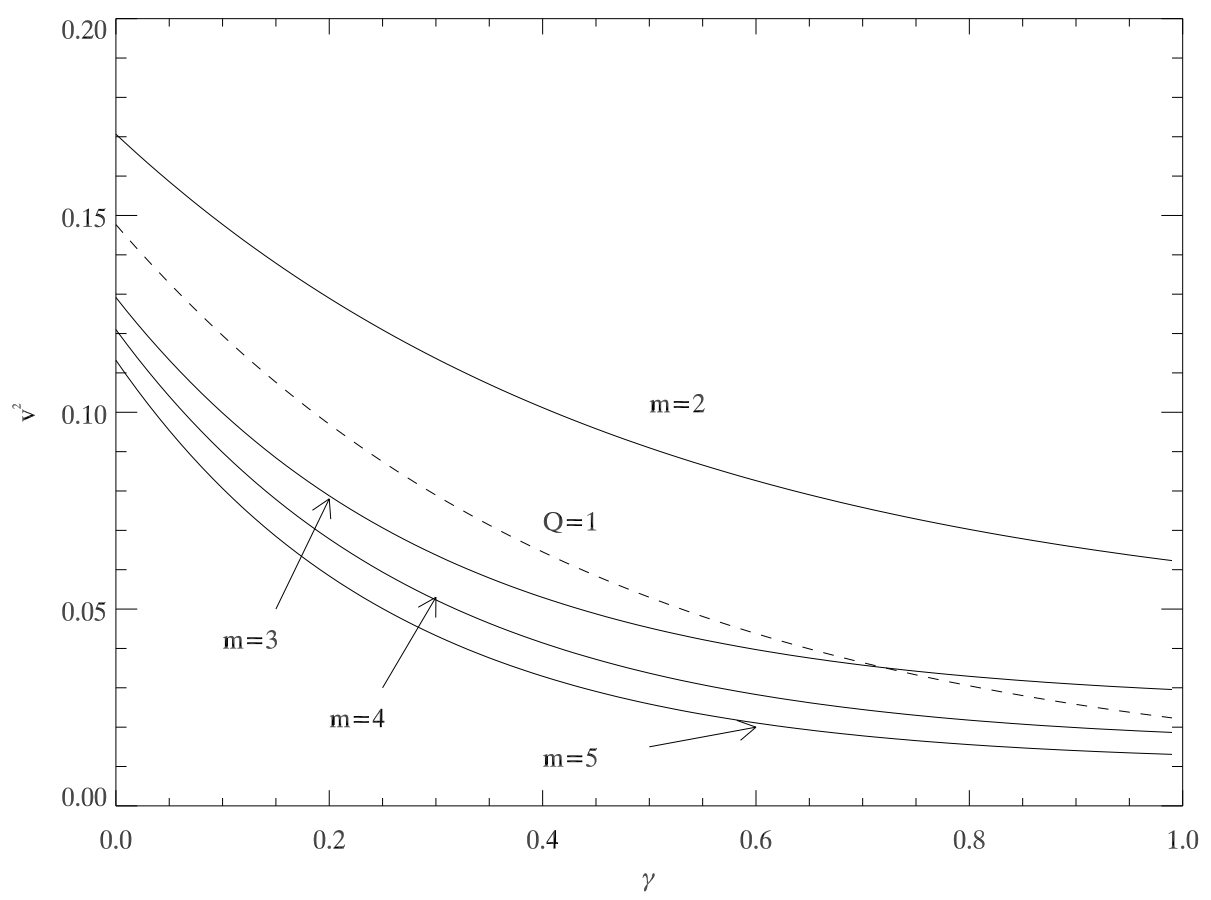

Fig. 1.- Radiation driven neutral modes. For large enough values of $m$, the onset of instability is believed to occur at infinitesimal velocities. 


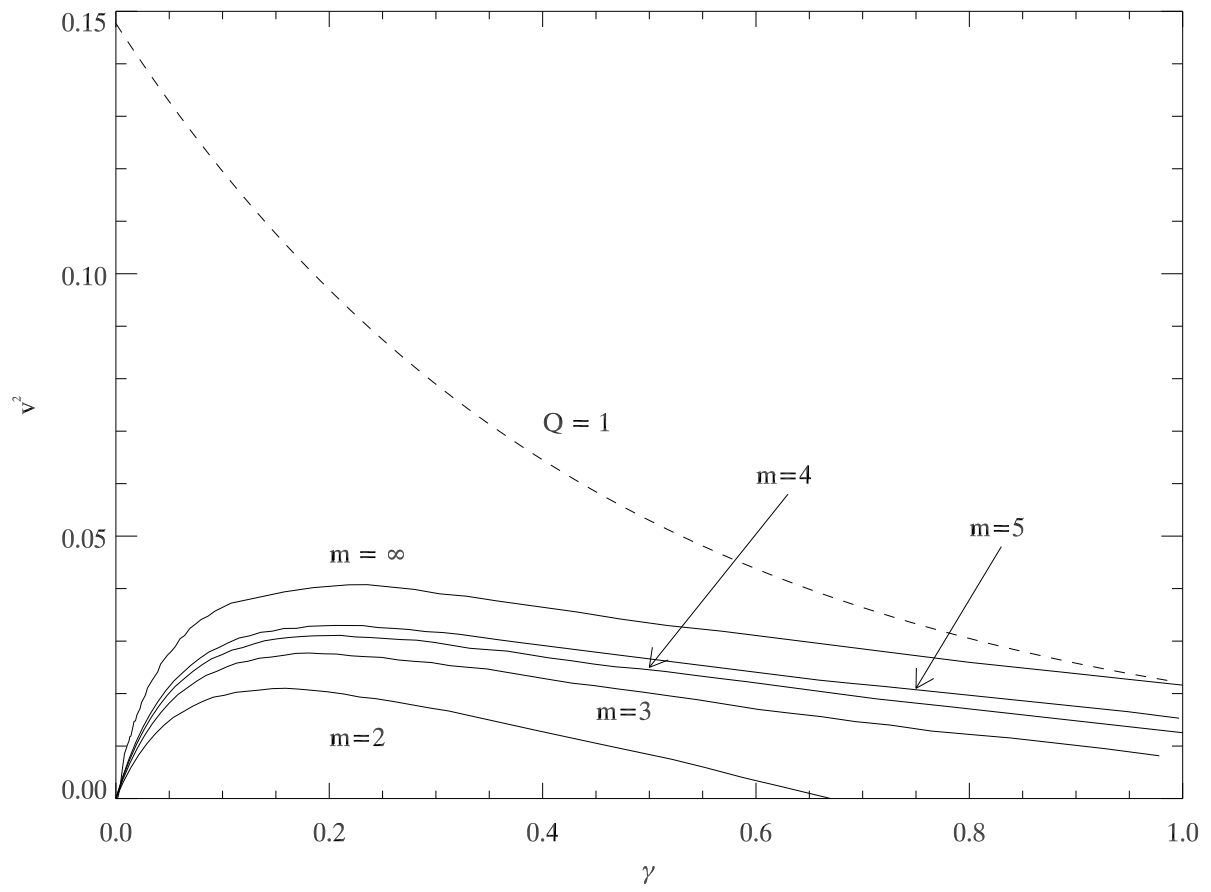

Fig. 2.- The neutral mode curves which are connected to the Newtonian bifurcation. In the lower-left corner, where $\gamma \rightarrow 0$ and $v \rightarrow 0$, the slope is $m /(m+2)$, as expected from the Newtonian limit. The dashed line separates the solution space into ergoregion and nonergoregion. 